\title{
Validation of an automated online 24-hour recall (myfood24) using nutrient biomarkers provides similar results to a traditional interviewer administered recall
}

\author{
Janet Cade $^{1}$, Petra Wark ${ }^{2}$, Gary Frost ${ }^{3}$, Nisreen Alwan ${ }^{4}$, Michelle Carter ${ }^{1}$, Paul Elliott ${ }^{3}$, \\ Heather Ford $^{3}$, Neil Hancock ${ }^{1}$, Michelle Morris ${ }^{1}$, Zeinab Mulla ${ }^{3}$, Essra Noorwali ${ }^{1}$, \\ Aikaterini Petropoulou ${ }^{3}$, Greg Potter ${ }^{1}$, Elio Riboli ${ }^{3}$, Laura Hardie ${ }^{1}$ and Darren Greenwood ${ }^{1}$ \\ ${ }^{1}$ University of Leeds, Leeds, United Kingdom, \\ ${ }^{2}$ Coventry University, Coventry, United Kingdom, \\ ${ }^{3}$ Imperial College, London, United Kingdom and \\ ${ }^{4}$ University of Southampton, Southampton, United Kingdom
}

\begin{abstract}
Online dietary assessment tools can reduce administrative costs and facilitate repeated dietary assessment during follow-up in large-scale prospective studies. We developed an online 24-h recall (myfood24) with automated estimation of associated nutrient intake, and assessed validity against reference recovery, predictive and concentration biomarkers. Validity of the online tool was then compared with that of traditional interviewer-administered multiple-pass 24-h recalls and presented as the expected attenuation of any diet-disease associations estimated with the tool.

Metabolically stable adults were recruited and completed the new online dietary recall, a traditional interviewer-based multiple-pass recall and provided samples of blood and urine for a range of reference biomarkers. Longer-term dietary intake was estimated from up to three recalls taken two weeks apart. Estimated intakes of protein, total sugars, potassium and sodium were compared with urinary biomarker concentrations. Estimated energy intake was compared with energy expenditure measured by three-plane accelerometry and open-circuit indirect calorimetry. Validity against these biomarkers was also compared to that estimated for traditional interviewer-administered multiple-pass 24-hour recalls.

At least one biomarker sample was received from each of 212 participants. Compared to reference biomarkers, both the online 24-hour recall and interviewer-based recall led to attenuation of diet-disease associations. The online tool resulted in attenuation factors of around 0.2-0.3 which could have important effects on estimated risks. For example, if the true relative risk of a diet-disease association was 2.0, an attenuation factor of 0.3 would reduce the relative risk to 1.23 . Ranking using intakes against repeated biomarkers as an estimate of truth, resulted in higher attenuation factors of approximately $0.3-0.4$, with a smaller impact on risk estimates. Attenuation improved substantially on repeated application of the tool. Validity of the interviewer-based recall found similar attenuation factors, but it was more administratively burdensome and expensive to implement. The online tool typically provided 10 $20 \%$ lower nutrient estimates compared to the interviewer-administered tool.

Our findings show that, whilst results from both automated online and traditional interviewer-based dietary recalls are attenuated compared to objective biomarker measures, the myfood 24 online 24 -hour recall is comparable to the more time-consuming and costly traditional interviewer-based 24-hour recall across a wide range of measures. The less burdensome implementation of the online tool, with automated nutrient coding and easy replication over a longer time period with associated gains in precision, makes it well-placed for repeated use in large-scale prospective studies.
\end{abstract}

\section{Conflict of Interest}

Prof Cade is a Director of University of Leeds spin out company Dietary Assessment Ltd, supporting myfood24. M Carter, N Hancock and M Morris are shareholders. 